\title{
Comprehensive metabolomics to evaluate the impact of industrial processing on the phytochemical composition of vegetable purees
}

\author{
Patricia Lopez-Sanchez ${ }^{\text {a,*,1 }}$, R.C.H. de $\operatorname{Vos}^{\text {b,e,f,1 }}$, H.H. Jonker ${ }^{\text {be,f, }}$, R. Mumm ${ }^{\text {b,f }}$, R.D. Hall ${ }^{\text {b,e,f }}$, L. Bialek ${ }^{\text {a }}$, \\ R. Leenman ${ }^{\text {a }}$, K. Strassburg ${ }^{\mathrm{d}, \mathrm{e}}$, R. Vreeken ${ }^{\mathrm{d}, \mathrm{e}}$, T. Hankemeier ${ }^{\mathrm{d}, \mathrm{e}}$, S. Schumm ${ }^{\mathrm{a}}$, J. van Duynhoven ${ }^{\mathrm{a}, \mathrm{c}, \mathrm{e}, \mathrm{C}^{\prime}}$ \\ ${ }^{a}$ Unilever Research and Development, Olivier van Noortlaan 120, 3133 AT Vlaardingen, The Netherlands \\ ${ }^{\mathrm{b}}$ Plant Research International, Wageningen University and Research Centre, Droevendaalsesteeg 1, 6708 PB Wageningen, The Netherlands \\ ${ }^{c}$ Laboratory of Biophysics, Wageningen University and Research Centre, Dreijenlaan 3, 6703 HA Wageningen, The Netherlands \\ ${ }^{\mathrm{d}}$ Leiden University, Einsteinweg 55, 2333 CC Leiden, The Netherlands \\ ${ }^{\mathrm{e}}$ Netherlands Metabolomics Centre, Einsteinweg 55, 2333 CC Leiden, The Netherlands \\ ${ }^{\mathrm{f}}$ Centre for Biosystems Genomics, P.O. Box 98, 6708 PB Wageningen, The Netherlands
}

\section{A R T I C L E I N F O}

\section{Article history}

Received 13 March 2014

Received in revised form 11 July 2014

Accepted 14 July 2014

Available online 24 July 2014

\section{Keywords:}

Metabolomics

Vitamins

Tomato

Broccoli

Carrot

Puree

Processing

LC-MS

GC-MS

NMR

LC-MRM MS

\begin{abstract}
A B S T R A C T
The effects of conventional industrial processing steps on global phytochemical composition of broccoli, tomato and carrot purees were investigated by using a range of complementary targeted and untargeted metabolomics approaches including LC-PDA for vitamins, ${ }^{1} \mathrm{H}$ NMR for polar metabolites, accurate mass LC-QTOF MS for semi-polar metabolites, LC-MRM for oxylipins, and headspace GC-MS for volatile compounds. An initial exploratory experiment indicated that the order of blending and thermal treatments had the highest impact on the phytochemicals in the purees. This blending-heating order effect was investigated in more depth by performing alternate blending-heating sequences in triplicate on the same batches of broccoli, tomato and carrot. For each vegetable and particularly in broccoli, a large proportion of the metabolites detected in the purees was significantly influenced by the blending-heating order, amongst which were potential health-related phytochemicals and flavour compounds like vitamins $C$ and E, carotenoids, flavonoids, glucosinolates and oxylipins. Our metabolomics data indicates that during processing the activity of a series of endogenous plant enzymes, such as lipoxygenases, peroxidases and glycosidases, including myrosinase in broccoli, is key to the final metabolite composition and related quality of the purees.
\end{abstract}

(c) 2014 Elsevier Ltd. All rights reserved.

\section{Introduction}

A high intake of fruit and vegetables is recommended by health authorities worldwide due to a potential relationship with a reduced risk of cancer and cardiovascular diseases (Crowe et al., 2011; Leenders et al., 2013). Led by the consumers demand for more natural and healthier products, the food industry aims to (re)design manufacturing processes for improved nutritional quality of products based on fruit and vegetables. Carrot, tomato and broccoli are good sources of antioxidant vitamins (Steinmetz \& Potter, 1996), next to many other phytochemicals that are

* Corresponding author. Present address: ARC Centre of Excellence in Plant Cell Walls, CNAFS, The University of Queensland, St. Lucia 4072, Queensland, Australia. Tel.: +61 733460793; fax: +61 733650539 .

E-mail addresses: p.lopezsanchez@uq.edu.au (P. Lopez-Sanchez), john.van. duynhoven@wur.nl (J. van Duynhoven).

1 Both authors contributed equally to this work.

2 Tel.: +31 317482044; fax: +31317482725. potentially healthy for human, like various flavonoids and phenylpropanoids (Moco et al., 2006; Vallejo, Tomas-Barberan, \& Ferreres, 2004). Broccoli also contains considerable amounts of glucosinolates, a group of phytochemicals specifically found in brassica vegetables (Moreno, Carvajal, Lopez-Berenguer, \& Garcia-Viguera, 2006).

During food manufacture phytochemicals may undergo thermal degradation, air exposure due to disruption of plant tissues/cells and endogenous enzymatic activity. For example, vitamin $\mathrm{C}$ can be easily lost, especially during cooking, due to its sensitivity to heat (Francisco, Velasco, Moreno, Garcia-Viguera, \& Cartea, 2010). Semi-polar phytonutrients like flavonoids and glucosinolates can also be lost upon heating vegetables (Aires, Carvalho, \& Rosa, 2012; Francisco et al., 2010). In contrast, there is generally a relatively high retention of hydrophobic compounds such as $\beta$-carotene during thermal treatments (Svelander, Lopez-Sanchez, Pudney, Schumm, \& Alminger, 2011).

Furthermore, not only the retention but also the bioaccessibility of health related compounds can be influenced by 
processing conditions due to the different microstructures which can be generated (Parada \& Aguilera, 2007). For example, the in vitro bio-accessibility of lycopene from tomato can be increased by thermal and mechanical processing, as compared to unprocessed fruit (Tiback et al., 2009). Likewise, it has been shown that shear treatments to disrupt plant tissues/cells, such as high pressure homogenisation ( $\mathrm{HPH})$, can increase the bio-accessibility of carotenes from both tomato and carrot (Svelander et al., 2011). Thus, food processing may be optimised to enhance both the content and the bio-accessibility of phytonutrients present in final food products based on fruit and vegetables (Sanchez-Campillo et al., 2012).

It is still a considerable challenge for industries to design food manufacturing processes which provide products with the desired balance between nutritional and sensorial quality. A bottleneck in the design of such processes is the lack of insight into the impact of processing steps on the overall metabolite profile of fruits and vegetables. Metabolomics is a powerful tool to generate comprehensive insights into the metabolic composition of crude extracts and is increasingly being applied in food science (Beleggia et al., 2011; Capanoglu, Beekwilder, Boyacioglu, Hall, \& De Vos, 2008; Hall, Brouwer, \& Fitzgerald, 2008; McGhie \& Rowan, 2012). A number of targeted metabolomics studies have been carried out to evaluate the impact of different processing conditions and steps on a specific class of phytochemicals, such as carotenoids in paprika paste (Schweiggert, Kurz, Schieber, \& Carle, 2007) and glucosinolates in broccoli (Vallejo, TomasBarberan, \& Garcia-Viguera, 2002), whereas large-scale untargeted metabolomics approaches have been applied to determine the global changes occurring upon processing of, for instance, tomatoes towards tomato paste (Capanoglu et al., 2008) and grain towards pasta (Beleggia et al., 2011).

In the present study, the effect of changing the order of thermal and mechanical processing steps on the phytonutrient profiles of carrot, tomato and broccoli purees was investigated, using a comprehensive metabolomics approach. Our objective was to evaluate the effect of different processing conditions on the phytonutrient profiles of the resulting vegetable purees. Experiments were therefore carried out on the same batch of material, to exclude biological variation between starting materials. We deployed both targeted analyses platforms, focussed on known health-related antioxidants, such as carotenes and vitamins $\mathrm{C}$ and $\mathrm{E}$, and oxidised lipids, as well as a suite of complementary untargeted metabolomics platforms, including ${ }^{1} \mathrm{H}$ NMR for polar metabolites, accurate mass LC-QTOF MS for semi-polar metabolites and headspaceGC-MS for volatile compounds.

\section{Material and methods}

\subsection{Materials}

Three plant materials were selected due to the fact that they are major crops and they are present in many plant-based food products: carrots (Daucus carota) as an example of a root vegetable, broccoli (Brassica oleracea) as a stem vegetable and red ripe tomatoes (Solanum lycopersicum) as a fruity vegetable. They were purchased from a local supplier (Hofland, The Netherlands) and immediately processed.

The origin of all the chemicals and standards used in the various analytical methods are described in the related references. Sulforaphane was purchased from Sigma Aldrich.

\subsection{Processing conditions}

Carrot, broccoli and tomato were washed and cut into pieces of approximately $2 \mathrm{~cm}$ in size. The carrots were peeled prior to washing. The large stems of the broccoli and the cores of the tomato fruits were removed. Per vegetable, the pieces were randomly mixed into a single batch, of approximately $2 \mathrm{~kg}$, to reduce variability from individual specimens and this batch was then divided into two sets, which were either first mechanically disrupted using a kitchen blender and then heated (B-T samples) or first heated and then blended (T-B samples). Pieces were mixed with deionised water, to facilitate the blending and homogenisation steps, in a ratio vegetable: water of $1: 1$ for carrot and broccoli, and 9:1 for tomato in view of the high amount of free water present in this fruit. One set of vegetable pieces (B-T) was firstly mechanically disrupted using a blender (Model CBT500E, Cuisinart $^{\circledR}$, East Windsor, NJ, USA) at maximum speed for 3 min. A sample of each of the resulting slurries was taken as the non-heated control (blended only; B) and the remainder was heated using a water bath. In the preliminary experiment we applied two conditions: $70 \pm 5^{\circ} \mathrm{C}$ for $10 \mathrm{~min}$ as a mild blanching (T1) and $90 \pm 5^{\circ} \mathrm{C}$ for $40 \mathrm{~min}$ as a more extreme cooking step (T2). The samples were covered with aluminium foil to prevent evaporation, as it was determined by weighing the samples before and after heating. The second set of pieces (T-B) was first heated in the same amount of water as the first set, followed by blending. The temperature in the core of the pieces was monitored using two thermocouples (manufactured in house) attached to a data logger. The samples were gently stirred during heating. In the exploratory experiment we also included high pressure homogenisation as a more severe mechanical treatment (60 MPa, Panda $2 \mathrm{k}$ high pressure homogeniser, Niro Soavi, Parma, Italy). The processing conditions studied are representative of those thermal and shear treatments currently used in food industry to produce liquid and semi-liquid plantbased food products. Suppl. Fig. S1 summarises the combinations of the different processes.

For the large-scale comparative metabolomics study, both B-T2 and $\mathrm{T} 2-\mathrm{B}$ processing conditions were repeated 3 times on the same batch of raw material, in order to specifically focus on the effects of the differential order of processing and avoid inference from biological variation.

Irrespective of processing conditions, the resulting carrot, broccoli and tomato purees had a $\mathrm{pH}$ of $5.9,6.2$, and 4.3 , respectively. Immediately after processing, the samples were frozen in liquid $\mathrm{N}_{2}$ and ground to a fine powder, which was stored at $-80^{\circ} \mathrm{C}$ and kept frozen up to extraction into solvent for metabolite analyses.

\subsection{Targeted analyses of vitamin $C$, sulforaphane and lipid-soluble isoprenoids}

From each sample, $0.5 \mathrm{~g}$ frozen powder (FW) was weighed for extraction of lipid soluble isoprenoid compounds, including carotenoids, tocopherols and chlorophylls. Extraction and analysis was performed essentially as described previously (Capanoglu et al., 2008), using $2.5 \mathrm{ml}$ methanol, $2 \mathrm{ml}$ chloroform containing $0.1 \%$ BHT as antioxidant and $2.5 \mathrm{ml}$ of $50 \mathrm{mM}$ Tris-buffer $\mathrm{pH}$ 7.5. Sudan $1(27 \mu \mathrm{g})$ was used as internal standard. Puree samples were extracted with chloroform three times, thereby collecting all visible pigments from the samples. Chloroform fractions were pooled and dried under $\mathrm{N}_{2}$ gas, re-dissolved in $0.5 \mathrm{ml}$ of ethylacetate and subsequently analysed by HPLC equipped with both a photodiode array detector (PDA; 240-600 nm) for the analysis of carotenoids and chlorophylls, and a fluorescence detector for the analyses of tocopherols. Vitamin C (ascorbic acid) was extracted using metaphosphoric acid and analysed by HPLC-PDA (Capanoglu et al., 2008). Sulforaphane was analysed by GC-MS after extraction with dichloromethane (Chiang, Pusateri, \& Leitz, 1998). Authentic standards were used to construct calibration curves for compound quantification. Variation between replicate extractions and 
analyses of samples from each vegetable puree was less than $7 \%$ for all target compounds.

\subsection{Oxylipin profiling}

For targeted analyses of oxidised fatty acids, apolar extracts were prepared as described above for carotenoid profiling. Ten microlitre of a $1 \mathrm{mM}$ oxylipin mix (Strassburg et al., 2012) was added as internal standard. The dried chloroform extracts were reconstituted in $100 \mu \mathrm{L}$ of injection solvent containing acetonitrile and methanol $(1: 1 \mathrm{v} / \mathrm{v})$. The samples were analysed by liquid chromatography (Agilent 1260, San Jose, CA, USA) coupled to electrospray ionisation on a triple quadrupole MS (Agilent 6460, San Jose, CA, USA) as recently described (Strassburg et al., 2012). In short, chromatographic separation was achieved on an Ascentis Express $(2.1 \times 150 \mathrm{~mm}, 2.7 \mu \mathrm{m}$ particles; Supelco $)$ column using a flow rate of $0.35 \mathrm{ml} / \mathrm{min}$ at $40^{\circ} \mathrm{C}$ and a 28 min gradient. Oxylipins were detected in negative ionisation mode with specific multiple reaction monitoring within fixed retention time windows, determined using authentic standards. Peak area integration was performed with Mass Hunter Quan version B.04.00 (Agilent). The peak areas of target compounds were corrected for extraction and injection variation using their internal standard responses.

\subsection{Untargeted NMR profiling of polar metabolites}

Samples were subjected to untargeted profiling of polar metabolites by ${ }^{1} \mathrm{H}$ NMR (Kim, Choi, \& Verpoorte, 2010). Briefly, plant material was frozen in liquid nitrogen, ground using a blender and freeze-dried. Subsequently, $50 \mathrm{mg}$ of freeze-dried material was vortexed and ultrasonicated in a 50/50 mixture of $\mathrm{CD}_{3} \mathrm{OD}$ and $\mathrm{KH}_{2} \mathrm{PO}_{4}$ buffer in $\mathrm{D}_{2} \mathrm{O}(\mathrm{pH} 6.0$ ) containing $0.55 \mathrm{mM} \mathrm{3-(tri-}$ methylsilyl) proprionic-2,2,3,3,-d4 acid (TSP) as internal standard. After centrifugation, the supernatant was filtered and transferred to a $5 \mathrm{~mm}$ (o.d.) NMR cryo probe tube. One-dimensional ${ }^{1} \mathrm{H}$ NMR spectra were acquired on a Bruker Avance III $600 \mathrm{MHz}$ NMR spectrometer operating at a proton frequency of $600.13 \mathrm{MHz}$. The temperature was kept constant at $26.8^{\circ} \mathrm{C}$. Automatic tuning, matching and shimming were applied before acquisition. The $90^{\circ}$ pulse was determined for every sample. The spectra were acquired with presaturation of the water resonance using a noesy $1 \mathrm{dpr}$ pulse sequence RD-90 $0^{\circ}$-F1-90'-Fmix-90 ${ }^{\circ}$-FID (Bruker Biospin, Germany). Here, $\mathrm{F} 1$ is a $4 \mu$ s delay time, and Fmix is the mixing time $(150 \mathrm{~ms})$. The free induction decays (FIDs) were collected into $64 \mathrm{~K}$ points (128 scans) with a spectral width of $8499 \mathrm{~Hz}$, an acquisition time of $3 \mathrm{~s}$ and a relaxation delay of $30 \mathrm{~s}$. The spectra were phase- and baseline corrected using Topspin 1.3.4 software (Bruker, Rheinstetten, Germany). Metabolite levels were obtained by straightforward integration of assigned signals, using the signal of TSP as standard. The technical variation of the overall procedure (both extraction and NMR quantification) ranged between $1-6 \%$ and 3-7\% for high ( $>40 \mathrm{mmol} / 100 \mathrm{~g} \mathrm{DW})$ and low $(<90 \mu \mathrm{mol} / 100 \mathrm{~g} \mathrm{DW})$ metabolite levels, respectively. The limit of detection was $9 \mu \mathrm{mol} / 100 \mathrm{~g}$ DW.

\subsection{Untargeted LC-MS profiling of semi-polar compounds}

Frozen sample powder ( $0.5 \mathrm{~g} \mathrm{FW})$ was extracted in $75 \%$ aqueous methanol containing $0.1 \%$ formic acid, and analysed in an untargeted manner by reversed phase LC-PDA-QTOF MS in negative electrospray ionisation mode, using a $2.1 \times 150 \mathrm{~mm}$ Luna C18 column (Phenomenex), as described by De Vos et al. (2007). Raw data files were processed using Metalign software with a signal to noise threshold of 3 for baseline correction and peak picking. Aligned peak lists were then filtered for reproducible mass signals, i.e., present in at least 3 samples with an intensity of more than 6 times the noise. Redundant signals due to the presence of isotopes, in-source fragments and adducts were finally grouped into so-called reconstructed metabolites using MSClust software (Tikunov, Laptenok, Hall, Bovy, \& de Vos, 2012). To estimate the total technical variation of the entire workflow, 5 quality control samples (QCs) were prepared from a pooled puree sample and analysed and processed together with the individual puree samples. The average variation of the intensity of all reconstructed LC-MS metabolites detected in these QCs samples was $21 \%$. Selected compounds were manually annotated by matching the observed accurate masses (within $5 \mathrm{ppm}$ ) to plant metabolite databases, including the MotoDB (Moco et al., 2006), Komics DB (http:// webs2.kazusa.or.jp/komics), Metabolomics Japan DB (http:// metabolomics.jp) and the Dictionary of Natural Products (http://dnp.chemnetbase.com). Candidate structures were checked for corresponding in-source fragments and UV/Vis absorbance characteristics, if present, and for selected compounds by using commercially available authentic standards. Level of identifications was given according to the recommendations of the Metabolomics Society Initiative (Sumner et al., 2007).

\subsection{Volatile profiling by headspace SPME-GC-MS}

Vegetable puree samples were subjected to untargeted profiling of volatile compounds, using automated headspace solid-phase micro extraction (SPME) coupled to GC-MS (Tikunov et al., 2005). From each frozen and ground sample, $750 \mathrm{mg}$ FW was weighed into a $4 \mathrm{ml}$ glass vial with screw cap. After adding $750 \mu \mathrm{l} 100 \mathrm{mM}$ Na-EDTA pH 7.5 and $1.1 \mathrm{~g}$ solid $\mathrm{CaCl}_{2}$ (5 M final concentration) to the frozen powder to prevent enzymatic reactions, the vials were closed and sonicated for $10 \mathrm{~min}$. Then, $1 \mathrm{ml}$ of the puree- $\mathrm{CaCl}_{2}$ mixture was transferred into a $10-\mathrm{ml}$ crimp cap vial which was capped immediately. Volatiles were automatically extracted from the sample headspace using a $65 \mu \mathrm{m}$ PDMS/ DVB SPME fibre (Supelco) and injected into the GCMS (Fisons Instruments) via a Combi PAL autosampler (CTC Analytics). Untargeted data processing was performed using Metalign software followed by MSClust tool to reconstruct metabolites with their mass spectra (Tikunov et al., 2012). Reconstructed mass spectra of compounds were batch-wise matched with available databases containing mass spectra and retention indexes (NIST08, Wiley and an in-house library of natural VOCs), using a dedicated in house script. The best matching compound with both its corresponding forward and reversed spectral library match factors and its reported retention index, if available, were retained. For selected compounds their automatic annotations were manually checked using the AMDIS software for spectra deconvolution and matching their observed retention index with reported retention index data, if available. Total technical variation of this platform was $25 \%$, as was estimated from QC samples extracted and analysed within the same sample series. Reconstructed volatiles with unreliable annotations, i.e., with a spectral match $<700$ and RI deviation $>50$ units, were removed from the resulting compound list. Level of identifications was given according to the recommendations of the Metabolomics Society Initiative (Sumner et al., 2007).

\subsection{Multivariate analyses and statistics}

GeneMaths XT software (Applied Maths, Belgium) was used for Principal Components Analysis. Signal intensities of reconstructed metabolites were log2-transformed and mean-centred over the samples. Univariate analysis (Students $t$-tests) was applied to determine metabolites significantly influenced by the processing order $(n=3)$ using a significance threshold of $\alpha=0.05$. 


\section{Results}

\subsection{Preliminary screen to select processing conditions for comparative metabolomics}

In order to select those vegetable processing conditions that can most severely influence the phytochemical profile of the purees, we performed a preliminary processing experiment (Suppl. Fig. S1) and analysed the resulting purees using both targeted analyses of health-related compounds (Suppl. Tables S1 and S2) and untargeted metabolomics profiling, using LC-QTOF MS, followed by an unsupervised principal components analysis (PCA) (Suppl. Fig. S3). In the latter analysis, in both broccoli and carrot purees the 1st PC coincided with the order of blending and heating (B-T versus T-B samples). In addition, in both broccoli and carrot purees that were blended without or prior to heating the levels of vitamin $C$ were low compared to purees that were heated before blending; this was also the case for vitamin E in broccoli (Suppl. Fig. S2). From this initial experiment we concluded that the phytochemical composition of the purees was mostly influenced by the order of blending and heating.

\subsection{Comparative metabolomics of thermal and blending treatment order}

We subsequently aimed to study the effect of changing the order of thermal and blending steps in a more detailed and statistically sound manner. In order to solely focus on the effect of processing order, the same starting material was used for each processing sequence, thereby minimising biological variation. Purees from carrot, broccoli and tomato were prepared in triplicate by applying three sequences of blending followed by heating at $90{ }^{\circ} \mathrm{C}$ for $40 \mathrm{~min}$ (B-T2) and firstly heating followed by blending (T2-B). The more extreme thermal treatment T2 was selected over $\mathrm{T} 1 \mathrm{as}$ it better resembles the temperature in the thermal treatments currently used by food industries. Resulting purees were subsequently subjected to comparative metabolomics, using a series of both targeted and untargeted profiling approaches, in order to determine the significant differences $(p<0.05)$ between both treatments $(n=3)$ in their effects on the phytochemical profiles of the products.

Quantitative, targeted analyses of selected phytochemicals (Suppl. Table S2, tab targeted analyses) indicated that for each vegetable the amount of vitamin $C$ was significantly lower in B-T2 purees than in T2-B purees. The most pronounced effect was observed in broccoli (27-fold difference, $p<0.001$ ) (Fig. 1), followed by carrot (factor $5, p=0.001$ ) and tomato (factor 3 , $p=0.001$ ). Sulforaphane, a glucosinolate-derived compound in broccoli, was detectable in B-T2 purees only (Suppl. Table S2, tab targeted analyses). Also in broccoli, all apolar isoprenoids analysed, i.e., tocopherols and carotenoids, were significantly ( $p$-values $<0.002$ ) lower in B-T2 compared to T2-B purees, ranging from $-31 \%$ for lutein up to $-60 \%$ for $\gamma$-tocopherol (Fig. 1). Likewise, in both carrot and tomato purees some but not all apolar isoprenoids were significantly lower in B-T2 puree: vitamin E was $18 \%$ and $19 \%$ lower and lutein $21 \%$ and $47 \%$ lower in carrot and tomato, respectively, as compared to their respective T2-B purees (Suppl. Table S2). In broccoli the level of $\beta$-carotene was $41 \%$ lower in B-T2 compared to T2-B puree, whilst in both carrot $(+17 \%)$ and tomato $(+18 \%)$ this compound was slightly but significantly higher in B-T2 than in the T2-B purees (Suppl. Table S2). These data suggest that the effect of blending-heating order on $\beta$-carotene level is dependent upon the vegetable matrix, whilst its effect on the vitamins $C$ and $E$ and on lutein is consistent between these three vegetables.

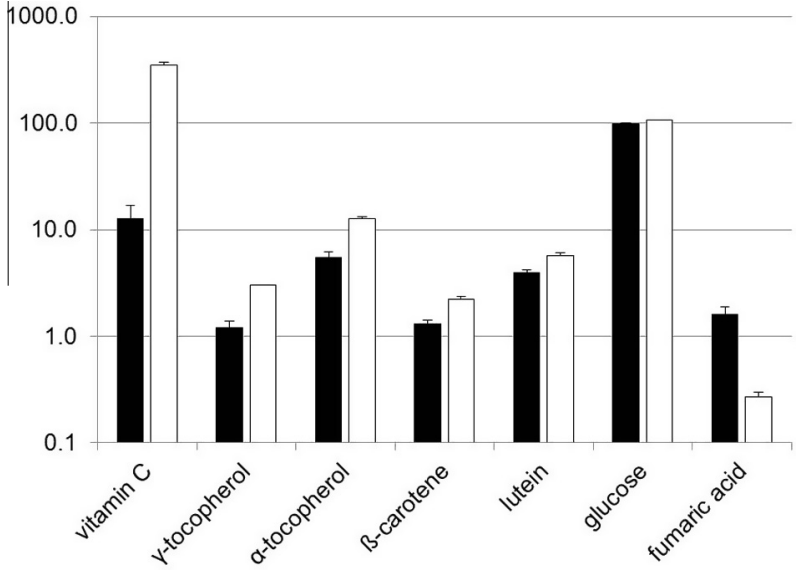

Fig. 1. Quantified metabolites in broccoli puree significantly $(p<0.05$, Student $t$ test) influenced by the heating-blending order. Black bars represent first blending then heating (B-T2) whilst white bars represent first heating then blending (T2-B). Standard deviations ( $n=3$ processing sequences) for each compound are indicated with error bars. Levels are in $\mathrm{mg} / \mathrm{g}$ DW (glucose and fumaric acid) or $\mathrm{mg} / \mathrm{kg} \mathrm{FW}$ (other metabolites). Note that $y$-axis is on a logarithmic scale.

The impact of heating-blending order on the global phytochemical composition of purees was further investigated using complementary, essentially untargeted profiling platforms. Polar primary metabolites were analysed by ${ }^{1} \mathrm{H}$ NMR profiling of aqueous extracts. In this NMR experiment, only two out of the three processing replicates were analysed. The NMR spectra obtained (Suppl. Fig. S4) indicated that the core of primary metabolites (sugars, organic acids) is shared between the three vegetables whilst their relative concentrations vary. The obtained spectral resolution of the NMR used allowed the quantification of 22 metabolites (Suppl. Table S2, tab targeted analyses). In broccoli, only fumaric acid (5.9-fold higher in B-T2) and glucose (7\% higher in B-T2) were significantly different between B-T2 and T2-B purees (Fig. 1). In carrot fumaric acid (4-fold) and guanoside (2-fold) were higher, whilst malic acid (4\%) and alanine (35\%) were lower in B-T2 versus T2-B purees. In tomato, only adenosine was slightly higher (10\%), whilst sucrose (10-fold), alanine (39\%), $\gamma$-aminobutyric acid (22\%) and trigonelline (14\%) were significantly lower in B-T2 versus T2-B purees (Suppl. Table S2, tab targeted analyses).

The untargeted LCMS profiling, using a QTOF MS in negative ESI mode, of aqueous-methanol extracts of the purees was applied to determine the effects on the relative levels of semi-polar, mostly secondary metabolites (Suppl. Table S2, tab untargeted LCMS). The differential LCMS chromatograms of broccoli (Fig. 2) show that metabolites from different biochemical classes, including glucosinolates, flavonoids and oxylipins, were influenced by the heatingblending order. In broccoli, the B-T2 treatment resulted in highly significant $(p<0.001)$ lower signals of all glucosinolates as compared to T2-B (Fig. 2 and Suppl. Table S2). The largest differences were observed for 3-indolylmethyl-glucosinolate (glucobrassicin, nearly 300 times lower), 1-methoxy-3-indolylmethylglucosinolate (neoglucobrassicin, 77 times lower) and 4-methylsulfinylbutylglucosinolate (glucoraphanin, 47 times lower), whilst the other glucosinolates were below detection limit after B-T2 treatment (Suppl. Table S2). Likewise, the B-T2 treatment resulted in significantly lower levels of phenylpropanoids like chlorogenic acid (4.5 fold, $p=0.001$ ) and sinapoyl-glucoside ( 2 fold, $p=0.003$ ), as well as of both UDP-glucose and UDP-N-acetyl-glucosamine (both undetectable in B-T2) and of the antioxidant glutathione (23-fold lower). On the other hand, several compounds with their accurate masses corresponding to hydroxylated fatty acids (oxylipins) were 3-47 fold higher in B-T2 purees than in T2-B purees. Other 

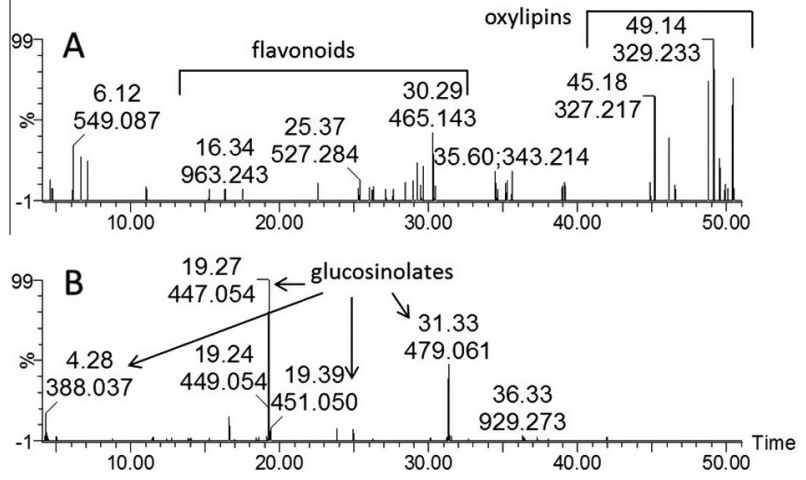

Fig. 2. Effect of blending-heating order on semi-polar metabolites in broccoli puree. Crude aqueous-methanol extracts were subjected to untargeted profiling using C18-reversed phase LC-QTOF MS (in ESI negative mode), and Metalign software was used to extract all mass signals above 3 times the noise and pinpoint those signals that are significantly ( $p<0.01, n=3$ ) at least 2-fold higher (A) or lower (B) in B-T2 (Blending, Heating) versus T2-B (Heating, Blending) purees. Peaks are annotated with retention time ( $\mathrm{min}$ ) and accurate mass and peak heights represent mean relative differences between groups. Metabolite classes mainly affected are indicated by boxes or arrows. See Suppl. Table S2 for info of all LCMS compounds.

compounds significantly higher in B-T2 included kaempferolconjugates (up to 3 fold), guanosine-phosphate ( 4 fold), cyclic GMP (14 fold) and S-sulphoglutathione (19 fold) (Suppl. Table S2, tab untargeted LCMS).

In order to confirm the increase of oxylipins observed in the untargeted LCMS approach, we deployed a dedicated LC-MRM MS platform enabling the detection and quantification of a wide range of oxylipin species (Strassburg et al., 2012). In all vegetables the B-T2 purees contained relative high levels of several oxylipins, as compared to their T2-B purees (Suppl. Table S2, tab Targeted analyses). In B-T2 samples of both carrot and broccoli, 13S-hydroxy-9Z,11E-octadecadienoic acid (13-HODE) and 9S,12S,13S-trihydroxy-10E-octadecenoic acid (9,12,13-TriHOME) were the major oxylipin species, whilst in tomato also 9S-hydroxy-10E,12Z-octadecadienoic acid (9-HODE) and 9-keto-

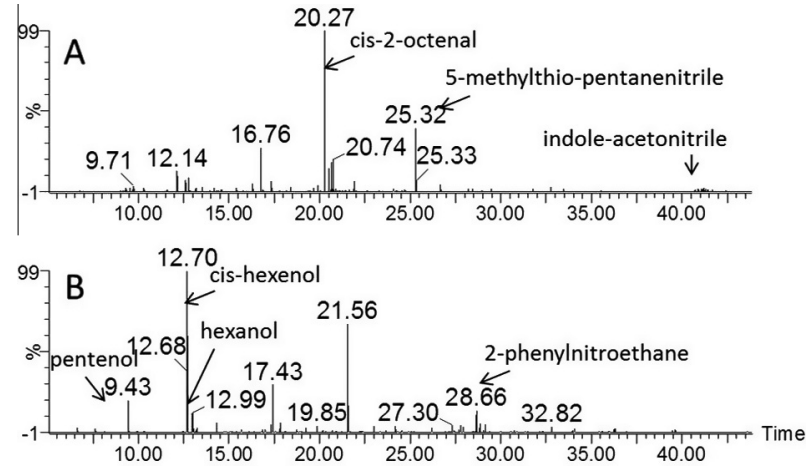

Fig. 4. Effect of blending-heating order on the volatile profiles of broccoli purees. Untargeted headspace SPME-GCMS and Metalign software was used to extract all mass signals above 3 times the noise and pinpoint those signals that are significantly $(p<0.05)$ at least 2-fold higher (A) or lower (B) in B-T2 (Blending, Heating) versus T2-B (Heating, Blending) purees. Peaks are annotated with retention time $(\mathrm{min})$, whilst peak heights represent mean relative differences between groups. Annotations of selected metabolites are indicated. See Suppl. Table S2 for info of all compounds detected.

10E, 12Z-octadecadienoic acid (9-KODE) contributed considerably to the total oxylipin level. The largest differences between B-T2 and T2-B in oxylipins were observed in broccoli (Fig. 3) and the smallest in carrot. On the other hand, the highest number of differential oxylipins was detected in tomato (Suppl. Table S2).

Using SPME-headspace trapping followed by untargeted GCMS profiling, we also determined the effect of the order of blending and thermal treatments on differences in volatiles derived from the purees. For each vegetable, a large percentage of the detectable volatile compounds appeared differential $(p<0.05)$ between B-T2 and T2-B purees (Suppl. Table S2), with again the largest effect in broccoli (Fig. 4). From the 269 volatiles detected in broccoli, 128 (i.e., $48 \%$ ) were significantly different, of which 62 (23\% of total) were at least 2-fold lower and 39 (i.e., $14.5 \%$ ) at least 2-fold higher in B-T2 compared to T2-B purees. Amongst the (putatively) annotated compounds that were lower in B-T2 samples were hexanoic

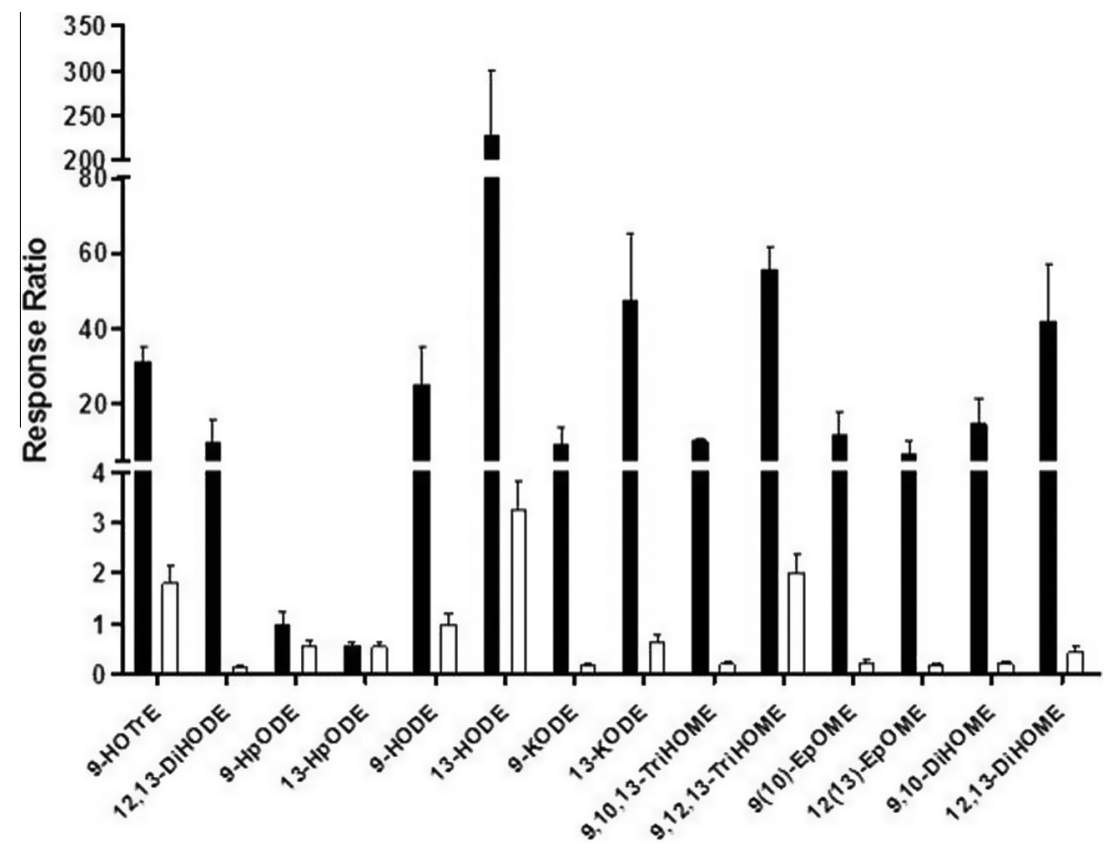

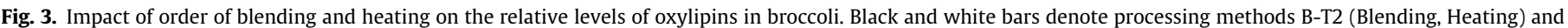

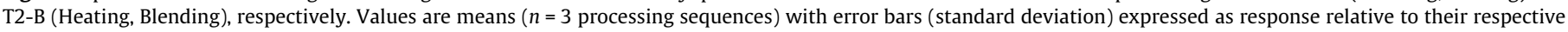
internal standards. See Suppl. Table 2 for full names of oxylipin species. 
acid (undetectable in B-T2 samples), several glucosinolate-derived volatiles such as indole-acetonitrile, indole-carbonitril and 5-methylthio-pentanenitrile (40-100 fold lower), as well as lipid oxidation-derivatives such as 1,5-octadien-3-ol and 2,6-nonadienal (about 5-fold lower). On the other hand, several other glucosinolate-derived volatiles, including dimethyldisulphide (5 fold), methyl thiocyanate (16 fold) and 2-ethyl thiophene ( 26 fold), but also lipid oxidation-derived C6-aldehydes like cis 3-hexen-1-ol (50 fold) and hexanol ( 73 fold), as well as isoprenoid-derived volatiles like beta-damascenone and gamma-cuprenene (both undetectable in T2-B samples) were significantly higher in B-T2 compared to T2-B broccoli purees. In carrot purees, $25 \%$ of the volatiles detected by SPME-GCMS were significantly different between treatment orders, of which $15 \%$ was at least 2 -fold changed, whilst in tomato purees these values were $26 \%$ and $18 \%$, respectively (Suppl. Table S2). These data clearly indicate large and consistent effects of the order of blending and thermal treatment on the volatile composition of the resulting purees.

\section{Discussion}

The processing treatments used in the present study were selected in view of their potential to be used to structure liquid and semiliquid foods derived from fruit and vegetables (Van Buggenhout et al., 2012). In order to get a rough indication of which processing treatments have most influence on the phytochemical profiles of food products, three commonly consumed vegetables, i.e., carrot, broccoli and tomato, were processed in 7 different manners (Suppl. Fig. S1) and the resulting purees were compared for their metabolite composition (Suppl. Figs. S2 and S3, and Table S1). This initial experiment indicated that the order of blending and heating mostly influences the final puree in both carrot, tomato and, in particular, broccoli. Microscopic analysis of the resulting purees (data not shown) agreed with the previously reported findings (Lopez-Sanchez et al., 2011), showing that the microstructure of the purees can be influenced by the order of thermal and shear treatments. In order to get more insight into the precise effect of changing the blending and heating order on the global phytochemical profile of the final products, we applied a multiplatform metabolomics approach, consisting of both targeted and untargeted analytical technologies, on purees of carrot, tomato and broccoli obtained by three independent processing sequences with alternating blending-heating order.

\subsection{Effect of heating-blending order on antioxidant compounds}

Vitamin C losses during food processing are likely to be due to a combination of (endogenous) enzymatic activity (e.g., ascorbic acid oxidase) and heat treatment. In all three vegetables the retention of vitamin $\mathrm{C}$ was better when the thermal treatment was performed on the roughly cut vegetable pieces before blending (T-B) than after blending (B-T). Likewise, the water-soluble antioxidant glutathione, a cysteine-containing tripeptide, was higher in all three vegetable purees if heated before blending (Suppl. Table S2). In broccoli, the B-T treatment also resulted in lower levels of lipid-soluble antioxidants, like vitamin E ( $\alpha$-tocopherol) and carotenoids, as compared to T-B purees. Likewise, lutein was lower in the B-T purees of all three vegetables. In contrast, $\beta$-carotene in carrot and both $\beta$-carotene and all-trans lycopene in tomato were up to $20 \%$ higher in B-T purees than in T-B purees, in line with literature showing that thermal and shear treatments have the potential to enhance the bio-accessibility of lipid-soluble compounds like carotenoids(Anese, Falcone, Fogliano, Nicoli, \& Massini, 2002; Svelander et al., 2011). Our results indicate that the effect of blending-heating order on the levels of lipid-soluble antioxidants is dependent upon carotenoid species as well as on vegetable matrix.

\subsection{Differential metabolomics reveals major effects of heating- blending order on metabolite profiles of purees}

The ${ }^{1} \mathrm{H}$ NMR profiling and quantification indicated minor effects on polar primary metabolites, except for a few compounds in the individual vegetables (Suppl. Table S2). For instance, blending before heating resulted in significantly lower levels of sucrose in tomato, likely due to endogenous invertase activity. On the other hand, the LCMS profiling indicated marked differences in some other amino acids and their derivatives in one or more vegetables. Amongst these was the tri-peptide antioxidant glutathione, which was significantly lower in B-T2 purees than in T2-B purees. Targeted analyses of the antioxidants vitamins $C$ en $E$ also indicated that in the B-T2 purees oxidation-related processes are enhanced. This conclusion is supported by the observed higher levels of several oxidised fatty acid species in the broccoli B-T2 samples, as detected by both the untargeted LC-QTOF MS profiling (Fig. 2) and the LC-MS/MS platform dedicated to oxylipin analyses (Fig. 3). The higher levels of oxylipins in B-T2 compared to T2-B purees coincided with an increase in the relative levels of lipid oxidation-related volatile C6-aldehydes, such as cis-3-hexenol, in all vegetables (Fig. 4 and Suppl. Table S2).

Processing treatments may result in flavonoid degradation when oxidative enzymes like polyphenol oxidases (Pilizota \& Subaric, 1998) have not been adequately inactivated by, for instance, heating. On the other hand, it has been observed that tomato fruit-breaking, i.e., cutting fresh tomatoes as the first step in processing, coincided with a two-fold increase in the level of the major quercetin-glycoside rutin, which was suggested to result from the activation of the fruit's wound response (Capanoglu et al., 2008). Comparison of the LCMS profiles of the vegetable purees analysed in the present study (Suppl. Table S2) indicated that in broccoli most of the flavonoid conjugates identified were significantly higher in B-T2 compared to T2-B samples. The higher level in B-T2 puree may thus indicate a wounding reaction of broccoli upon blending before subsequent enzyme inactivation by the heating step. In contrast, the main flavonoids in tomato purees, i.e., rutin, naringenin and quercetin-trisaccharide, did not show differences between blending and heating order (Suppl. Table S2). The antioxidative phenylpropanoid chlorogenic acid was present in each vegetable. In both carrot and broccoli this compound was significantly lower in B-T2 versus T2-B purees, as were other phenylpropanoids in broccoli, indicating loss of this class of compounds when enzymes are not inactivated prior to blending. This difference in chlorogenic acid was however not observed in tomato, thus demonstrating vegetable-dependent processing effects on phenolic compounds.

Glucosinolates, an important group of nitrogen-containing thioglycosides in brassica vegetables, can be hydrolysed to form isothiocyanates, nitriles and/or thiocyanates through the action of endogenous myrosinase (thioglucoside glucohydrolase) and other proteins upon disruption of the cellular integrity (Kissen, Rossiter, \& Bones, 2009). However, the end-products formed depend upon many factors such as temperature, $\mathrm{pH}$, metals and, most importantly, the precise structure and concentration of the glucosinolates present (Hanschen et al., 2012; Verkerk et al., 2009). Myrosinase is active at temperatures up to $50{ }^{\circ} \mathrm{C}$ whilst temperatures of $80^{\circ} \mathrm{C}$ up to $100^{\circ} \mathrm{C}$ are needed for complete inactivation (Van Eylen, Oey, Hendrickx, \& Van Loey, 2008). The significantly lower levels of glucoraphanin and other glucosinolates in the broccoli B-T2 samples, as compared to the T2-B samples, can thus be explained by differential myrosinase activity between both processing methods (Verkerk et al., 2009). There is growing 
evidence that a higher intake of glucosinolates, through a higher consumption of brassica vegetables, can help to reduce the risk of cancer (Wang et al., 2004). Especially the glucosinolate hydrolysis-derived isothiocyanates in broccoli, such as indol-3-carbinol (from glucobrassicin) and sulforaphane (from sulforaphanin), are presumed to be the main bioactive components in broccoli (Moreno et al., 2006). We observed several glucosinolate-derived compounds, e.g., sulforaphane and methyl thiocyanate, that were higher in B-T2 than in T2-B samples (Suppl. Table S2). Importantly, intact glucosinolates, mostly present in the T2-B purees, can also be hydrolysed by the colon microflora thereby producing additional bioactive products (Moreno et al., 2006).

\subsection{Flavour and fragrance components are also affected by the processing regime}

In addition to their health-related bioactivities, broccoli glucosinolate breakdown products are odour-active compounds (Krumbein, Schonhof, Brückner, Wender, \& Mikael Agerlin, 2006). Also other volatiles that have known flavour impact, including lipid oxidation products like hexanal and decadienal, monoterpenes like $\alpha$-pinene and myrcene, benzene compounds like benzaldehyde and eugenol, and carotenoid-breakdown products like $\alpha$-ionone, were differentially affected by the blending-heating order in one or more vegetables (Fig. 4 and Suppl. Table S2). Clearly, for many volatile compounds the effect of swapping the blending and heating order differed between the three vegetables, and for some volatiles (e.g., pentanal, heptanal and 1-penten-3-one) even opposite effects were observed, indicating significant vegetable matrix effects. From a commercial point of view, organoleptic, appearance and nutritional effects must be considered and hence a broad analysis, which also take complex matrix effects into account, is required to better understand the optimal conditions of processing.

\section{Conclusion}

Using complementary targeted and untargeted comparative metabolite profiling techniques we obtained a comprehensive insight into the effects of industrial processing methods on the metabolite composition of carrot, tomato and broccoli purees used in liquid and semiliquid fruit and vegetable products such as soups, sauces and beverages. Using a design where processing methods was carried out in three repetitions, significant differences were observed between samples by swapping the order of blending and heating steps. Antioxidant vitamins, carotenoids, flavonoids, glucosinolates, volatiles (including odour-active compounds) and oxylipins were mostly influenced, depending on the vegetable. However, in all vegetables the blending of samples prior to heating (B-T2) resulted in significantly lower levels of both vitamin C and glutathione, with concomitant higher levels of oxidised fatty acids and lipid oxidation-derived volatiles like hexanal, as compared to blending after heating (T2-B), thus indicating a higher degree of food oxidation. This comprehensive metabolomics exercise indicated that industrial processing of raw vegetables into purees can have strong impact on the phytochemical composition of the final product, mainly due to activation of various endogenous enzymes upon blending. Included here are products from the lipoxygenase, (per)oxidases, and glycosidases (including myrosinase in broccoli) pathways, next to diverse non-enzymatic chemical reactions. Due to variation in both enzyme activities and chemical vegetable matrix such as $\mathrm{pH}$, which can differ between vegetable species, but also to genotype, growth and post-harvest conditions, vegetables can be differentially affected by the same processing treatment and hence tailor-made approaches are required for specific products and blends. In addition, the observed variation in metabolite profiles of purees obtained by replicate, principally equal processing events, whilst using the same starting material, indicates that comprehensive metabolomics can help the food industry to optimise their processing treatments for obtaining a more constant product quality.

\section{Acknowledgements}

P.L.S., J.vD., L.B., S.L. and S.S. are grateful to the European Commission for its financial support of the Healthy Structuring project (FP6-02311). R.dV., R.H., H.J., K.S., R.V., T.H.. J.vD., were partially funded by the Netherlands Metabolomics Centre (NMC) and R.dV., R.M., H.J. and R.H. also by the Centre of Biosystems Genomics, which are both part of the Netherlands Genomics Initiative/ Netherlands Organization for Scientific Research.

\section{Appendix A. Supplementary data}

Supplementary data associated with this article can be found, in the online version, at http://dx.doi.org/10.1016/j.foodchem.2014. 07.076 .

\section{References}

Aires, A., Carvalho, R., \& Rosa, E. (2012). Glucosinolate composition of Brassica is affected by postharvest, food processing and myrosinase activity. Journal of Food Processing and Preservation, 36(3), 214-224.

Anese, M., Falcone, P., Fogliano, V., Nicoli, M. C., \& Massini, R. (2002). Effect of equivalent thermal treatments on the color and the antioxidant activity of tomato purees. Journal of Food Science, 67(9), 3442-3446.

Beleggia, R., Platani, C., Papa, R., Di Chio, A., Barros, E., Mashaba, C., et al. (2011). Metabolomics and food processing: From semolina to pasta. Journal of Agricultural and Food Chemistry, 59(17), 9366-9377.

Capanoglu, E., Beekwilder, J., Boyacioglu, D., Hall, R., \& De Vos, R. (2008). Changes in antioxidant and metabolite profiles during production of tomato paste. Journa of Agricultural and Food Chemistry, 56(3), 964-973.

Chiang, W. C. K., Pusateri, D. J., \& Leitz, R. E. A. (1998). Gas chromatography mass spectrometry method for the determination of sulforaphane and sulforaphane nitrile in broccoli. Journal of Agricultural and Food Chemistry, 46(3), 1018-1021.

Crowe, F. L., Roddam, A. W., Key, T. J., Appleby, P. N., Overvad, K., Jakobsen, M. U., et al. (2011). Fruit and vegetable intake and mortality from ischaemic heart disease: Results from the European prospective investigation into cancer and nutrition (EPIC)-heart study. European Heart Journal, 32(10), 1235-1243.

De Vos, R. C. H., Moco, S., Lommen, A., Keurentjes, J. J. B., Bino, R. J., \& Hall, R. D. (2007). Untargeted large-scale plant metabolomics using liquid chromatography coupled to mass spectrometry. Nature Protocols, 2(4), 778-791.

Francisco, M., Velasco, P., Moreno, D. A., Garcia-Viguera, C., \& Cartea, M. E. (2010). Cooking methods of Brassica rapa affect the preservation of glucosinolates, phenolics and vitamin C. Food Research International, 43(5), 1455-1463.

Hall, R. D., Brouwer, I. D., \& Fitzgerald, M. A. (2008). Plant metabolomics and its potential application for human nutrition. Physiologia Plantarum, 132(2), $162-175$.

Hanschen, F. S., Bauer, A., Mewis, I., Keil, C., Schreiner, M., Rohn, S., et al. (2012). Thermally induced degradation of aliphatic glucosinolates: Identification of intermediary breakdown products and proposed degradation pathways. Journal of Agricultural and Food Chemistry, 60(39), 9890-9899.

Kim, H. K., Choi, Y. H., \& Verpoorte, R. (2010). NMR-based metabolomic analysis of plants. Nature Protocols, 5(3), 536-549.

Kissen, R., Rossiter, J. T., \& Bones, A. M. (2009). The 'mustard oil bomb': Not so easy to assemble?! Localization, expression and distribution of the components of the myrosinase enzyme system. Phytochemistry Reviews, 8(1), 69-86.

Krumbein, A., Schonhof, I., Brückner, B., Wender, L. P. B., \& Mikael Agerlin, P. (2006). Flavour and health-promoting compounds in broccoli and cauliflower-An inconsistency? Developments in food science (vol. 43, pp. 249-252). Elsevier.

Leenders, M., Sluijs, I., Ros, M. M., Boshuizen, H. C., Siersema, P. D., \& Ferrari, P. (2013). Fruit and vegetable consumption and mortality: European prospective investigation into cancer and nutrition. American Journal of Epidemiology.

Lopez-Sanchez, P., Nijsse, J., Blonk, H. C. G., Bialek, L., Schumm, S., \& Langton, M. (2011). Effect of mechanical and thermal treatments on the microstructure and rheological properties of carrot, broccoli and tomato dispersions. Journal of the Science of Food and Agriculture, 91(2), 207-217.

McGhie, T. K., \& Rowan, D. D. (2012). Metabolomics for measuring phytochemicals and assessing human and animal responses to phytochemicals, in food science. Molecular Nutrition \& Food Research, 56(1), 147-158.

Moco, S., Bino, R. J., Vorst, O., Verhoeven, H. A., de Groot, J., van Beek, T. A., et al (2006). A liquid chromatography-mass spectrometry-based metabolome database for tomato. Plant Physiology, 141(4), 1205-1218. 
Moreno, D. A., Carvajal, M., Lopez-Berenguer, C., \& Garcia-Viguera, C. (2006) Chemical and biological characterisation of nutraceutical compounds of broccoli. Journal of Pharmaceutical and Biomedical Analysis, 41(5), 1508-1522.

Parada, J., \& Aguilera, J. M. (2007). Food microstructure affects the bioavailability of several nutrients. Journal of Food Science, 72(2), R21-R32.

Pilizota, V., \& Subaric, D. (1998). Control of enzymatic browning of foods. Food Technology and Biotechnology, 36(3), 219-227.

Sanchez-Campillo, M., Larque, E., Gonzalez-Silvera, D., Martinez-Tomas, R., Garcia-Fernandez, M., Aviles, F. et al. (2012). Changes in the carotenoid concentration in human postprandial chylomicron and antioxidant effect in HepG2 caused by differently processed fruit and vegetable soups. Food Chemistry, 133(1), 38-44.

Schweiggert, U., Kurz, C., Schieber, A., \& Carle, R. (2007). Effects of processing and storage on the stability of free and esterified carotenoids of red peppers (Capsicum annuum L.) and hot chilli peppers (Capsicum frutescens L.). European Food Research and Technology, 225(2), 261-270.

Steinmetz, K. A., \& Potter, J. D. (1996). Vegetables, fruit, and cancer prevention: A review. Journal of the American Dietetic Association, 96(10), 1027-1039.

Strassburg, K., Huijbrechts, A. M. L., Kortekaas, K. A., Lindeman, J. H., Pedersen, T. L., Dane, A., et al. (2012). Quantitative profiling of oxylipins through comprehensive LC-MS/MS analysis: Application in cardiac surgery. Analytical and Bioanalytical Chemistry, 404(5), 1413-1426.

Sumner, L. W., Amberg, A., Barrett, D., Beale, M. H., Beger, R., Daykin, C. A., et al. (2007). Proposed minimum reporting standards for chemical analysis. Metabolomics, 3(3), 211-221.

Svelander C. A Lopez-Sanchez, P. Pudney, P. D. A Schumm, S., \& Alminger M. A. G. (2011). High pressure homogenization increases the in vitro bioaccessibility of alpha- and beta-carotene in carrot emulsions but not of lycopene in tomato emulsions. Journal of Food Science, 76(9), H215-H225.

Tiback, E. A., Svelander, C. A., Colle, I. J. P., Altskar, A. I., Alminger, M. A. G., Hendrickx, M. E. G., et al. (2009). Mechanical and thermal pretreatments of crushed tomatoes: Effects on consistency and in vitro accessibility of lycopene. Journal of Food Science, 74(7), E386-E395.

Tikunov, Y. M., Laptenok, S., Hall, R. D., Bovy, A., \& de Vos, R. C. H. (2012). MSClust: A tool for unsupervised mass spectra extraction of chromatography-mass spectrometry ion-wise aligned data. Metabolomics, 8(4), 714-718.

Tikunov, Y., Lommen, A., de Vos, C. H. R., Verhoeven, H. A., Bino, R. J., Hall, R. D., et al. (2005). A novel approach for nontargeted data analysis for metabolomics. Large-scale profiling of tomato fruit volatiles. Plant Physiology, 139(3), $1125-1137$.

Vallejo, F., Tomas-Barberan, F. A., \& Ferreres, F. (2004). Characterisation of flavonols in broccoli (Brassica oleracea L. var. italica) by liquid chromatography-UV diodearray detection-electrospray ionisation mass spectrometry. Journal of Chromatography A, 1054(1-2), 181-193.

Vallejo, F., Tomas-Barberan, F. A., \& Garcia-Viguera, C. (2002). Glucosinolates and vitamin $\mathrm{C}$ content in edible parts of broccoli florets after domestic cooking. European Food Research and Technology, 215(4), 310-316.

Van Buggenhout, S., Ahrné, L., Alminger, M., Andrys, A., Benjamin, M., Bialek, L., et al. (2012). Structural design of natural plant-based foods to promote nutritional quality. Trends in Food Science \& Technology, 24(1), 47-59.

Van Eylen, D., Oey, I., Hendrickx, M., \& Van Loey, A. (2008). Effects of pressure/ temperature treatments on stability and activity of endogenous broccoli (Brassica oleracea L. cv. Italica) myrosinase and on cell permeability. Journal of Food Engineering, 89(2), 178-186.

Verkerk, R., Schreiner, M., Krumbein, A., Ciska, E., Holst, B., Rowland, I., et al. (2009). Glucosinolates in Brassica vegetables: The influence of the food supply chain on intake, bioavailability and human health. Molecular Nutrition E' Food Research, 53, S219-S265.

Wang, L. I., Giovannucci, E. L., Hunter, D., Neuberg, D., Su, L., \& Christiani, D. C. (2004). Dietary intake of cruciferous vegetables, glutathione S-transferase (GST) polymorphisms and lung cancer risk in a Caucasian population. Cancer Causes $\mathcal{E}$ Control, 15(10), 977-985. 